\title{
Correction to: Peritoneal dialysis-related peritonitis caused by Gordonia bronchialis: first pediatric report
}

\author{
Valentina Bruno ${ }^{1,2}$ (D) James Tjon ${ }^{3} \cdot$ Sandy $\operatorname{Lin}^{4} \cdot$ Helen Groves $^{2,5} \cdot$ Kescha Kazmi $^{2,5} \cdot$ Michael Zappitelli $^{1,2}$. \\ Elizabeth Harvey ${ }^{1,2}$ [D
}

Published online: 10 January 2022

(c) The Author(s), under exclusive licence to International Pediatric Nephrology Association 2021

\section{Correction to: Pediatr Nephrol https://doi.org/10.1007/s00467-021-05313-3}

The final version of this article unfortunately contained a mistake. During typesetting process, the magnitude of the WBC reported in the abstract was erroneously rendered as WBC $\mathbf{2 , 3 4 0 \times 1 0 / L ~ ( 5 9 \% ~ n e u t r o p h i l s ) ~ a n d ~ G r a m - p o s - ~}$ itive bacilli. The correct magnitude, however, is WBC $\mathbf{2 , 3 4 0} \times \mathbf{1 0} / \mathbf{L}$ (59\% neutrophils) and Gram-positive bacilli. The publisher apologizes for this mistake. The original article has been corrected. The correct Abstract is presented below.

\section{Abstract \\ Introduction}

Gordonia species, aerobic, weakly acid-fast, Gram-positive bacilli, are a rare cause of peritonitis in patients undergoing peritoneal dialysis (PD). We report the first pediatric case of PD-related peritonitis caused by Gordonia bronchialis.

The original article can be found online at https://doi.org/10.1007/ s00467-021-05313-3.

Valentina Bruno

valentina.bruno@sickkids.ca

1 Division of Nephrology, The Hospital for Sick Children, 555 University Avenue, Toronto, ON M5G 1X8, Canada

2 Department of Paediatrics, University of Toronto, Toronto, ON, Canada

3 Department of Pharmacy, The Hospital for Sick Children, Toronto, ON, Canada

4 Department of Nursing, The Hospital for Sick Children, Toronto, ON, Canada

5 Division of Infectious Diseases, The Hospital for Sick Children, Toronto, ON, Canada

\section{Case presentation}

A 13-year-old girl with chronic kidney disease (CKD) stage 5D, on continuous cycling PD (CCPD) for 8 years, presented with cloudy PD effluent, with no abdominal discomfort or fever. Intra-peritoneal (IP) loading doses of vancomycin and ceftazidime were started at home after obtaining a PD effluent sample, which showed WBC $2,340 \times 10^{6} / \mathrm{L}$ (59\% neutrophils) and Gram-positive bacilli. On admission, she was clinically well and afebrile, with no history of methicillin-resistant Staphylococcus aureus (MRSA) infection, so vancomycin was discontinued, and IP ceftazidime and cefazolin were started, following a loading dose of intravenous cefazolin. Gordonia species grew after 5 days of incubation and later identified as Gordonia bronchialis. IP vancomycin was restarted as monotherapy, empirically for a total of 3 weeks therapy. A 2-week course of oral ciprofloxacin was added, based on susceptibility testing. PD catheter replacement was advised due to the risk of recurrence but was refused. A relapse occurred 16 days after discontinuing antibiotics, successfully treated with a 2-week course of IP ceftazidime and vancomycin. The PD catheter was removed and hemodialysis initiated. She received a further 2-week course of oral ciprofloxacin and amoxicillin-clavulanate post PD catheter removal.

\section{Conclusions}

Gordonia bronchialis is an emerging pathogen in PD peritonitis and appears to be associated with a high risk of relapse. PD catheter replacement is strongly suggested.

Publisher's note Springer Nature remains neutral with regard to jurisdictional claims in published maps and institutional affiliations. 